\title{
Partially ionized layers of accreted envelopes of weakly and strongly magnetized neutron stars
}

\author{
Alexander Potekhin ${ }^{1}$, Gilles Chabrier ${ }^{2}$, and Yuri Shibanov ${ }^{1}$ \\ ${ }^{1}$ Ioffe Physical-Technical Institute, 194021 St.-Petersburg, Russia \\ ${ }^{2}$ C.R.A.L., Ecole Normale Supérieure de Lyon, 69364 Lyon, France
}

\begin{abstract}
We study equilibrium properties of partially ionized hydrogen a.tmospheres and subphotospheric layers of weakly (with magnetic field $\left.B \ll 10^{9} \mathrm{G}\right)$ and strongly $\left(B \gg 10^{10} \mathrm{G}\right)$ magnetized neutron stars. In both weak- and strong-field cases, the ionization degree, atomic occupation numbers, and equation of state are calculated. These results are used to calculate opacities of neutron-star atmospheres.
\end{abstract}

Our study is aimed at construction of reliable atmosphere models and interpretation of the soft X-ray thermal emission of neutron stars with hydrogen atmospheres. Partial ionization, which occurs at temperature $T \lesssim 10^{5} \mathrm{~K}$ in the weak field and at $T \lesssim 10^{6.5} \mathrm{~K}$ in the strong field, affects the equation of state (EOS) and opacities. We study these effects using the "chemical picture" of the plasma (e.g., Saumon, Chabrier, \& Van Horn 1995). At $B=0$, we find a good agreement with the OPAL data (Iglesias \& Rogers 1996).

The motion of charged particles in a magnetic field is quantized into Landau orbitals. Free electrons are confined to the ground Landau level at temperatures $T \ll T_{B} \approx 10^{5.5} \gamma \mathrm{K}$ and densities $\rho<\rho_{B} \approx 0.809 \gamma^{3 / 2} \mathrm{~g} \mathrm{~cm}^{-3}$, where $\gamma \approx B /\left(2.35 \times 10^{9} \mathrm{G}\right)$ is the electron cyclotron energy in atomic units. In addition, the strong magnetic field $(\gamma \gg 1)$ drastically modifies the quantummechanical properties of hydrogen atoms and molecules. Thereby it affects the thermodynamics and radiative transport in the plasma.

Ionization equilibrium of hydrogen in strong magnetic fields was previously discussed by Gnedin, Pavlov, \& Tsygan (1974), Khersonskii (1987), and Lai \& Salpeter (1997). In our work, however, the modifications of atomic properties caused by thermal motion of the atoms across the field are properly taken into account for the first time. Namely, we use the results of quantum-mechanical calculations (Potekhin 1994, 1998) for a hydrogen atom in an arbitrary state of motion in the strong magnetic field. Details of our calculation of the EOS are published elsewhere (Potekhin, Chabrier, \& Shibanov 1999). Since the chemical picture provides atomic occupation numbers, it allows one to calculate the total opacities as a combination of the previously obtained opacities of the fully ionized plasma (Pavlov et al. 1995) and the absorption coefficients due to the boundbound (Pavlov \& Potekhin 1995) and bound-free (Potekhin \& Pavlov 1997) transitions. The figure illustrates the importance of the two latter components at the density, temperature, and magnetic field values typical for an atmosphere of a middle-aged pulsar. 

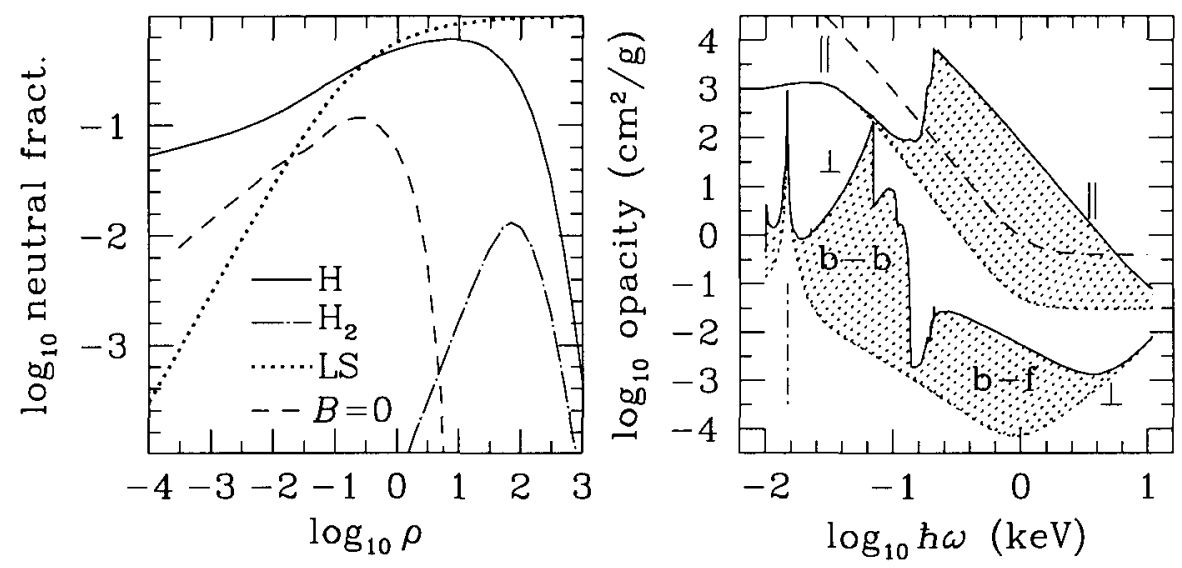

Figure 1. Role of the neutral fraction in a typical neutron-star hydrogen atmosphere at $B=2.35 \times 10^{12} \mathrm{G}$ and $T=3.16 \times 10^{5} \mathrm{~K}$.

Left: density dependence of atomic (solid line) and molecular (dotdashed line) fraction, compared with a simplified approximation of Lai \& Salpeter (1997, dotted line). Right: monochromatic opacity at $\rho=0.1 \mathrm{~g} \mathrm{~cm}^{-3}$ (solid line) for the polarization parallel $(\|)$ and perpendicular $(\perp)$ to the magnetic field. Shaded areas show contributions from bound-bound (b-b) and bound-free (b-f) radiative transitions. The dot-dashed line marks the proton cyclotron resonance. On both panels, dashed lines correspond to the zero-field case.

Acknowledgments. A.P. acknowledges hospitality of the theoretical astrophysics group at the Ecole Normale Supérieure de Lyon. This work was partially supported by INTAS Grant No. 96-542 and RFBR Grant No. 99-02-18099.

\section{References}

Gnedin, Yu. N., Pavlov, G. G., \& Tsygan, A. I. 1974, Sov. Phys. JETP, 39, 301 Iglesias, C. A., \& Rogers, F. J. 1996, ApJ, 464, 943

Khersonskii, V. K. 1987, Soviet Ast., 31, 225

Lai, D., \& Salpeter, E. E. 1997, ApJ, 491, 270

Pavlov, G. G., \& Potekhin, A. Y. 1995, ApJ, 450, 883

Pavlov, G. G., Shibanov, Yu. A., Zavlin, V. E., \& Meyer, R. D., 1995, in The Lives of the Neutron Stars, ed. M. A. Alpar, Ü. Kiziloğlu, \& J. van Paradijs (Dordrecht: Kluwer) 71

Potekhin, A. Y. 1994, J. Phys. B, 27, 1073

Potekhin, A. Y. 1998, J. Phys. B, 31, 49

Potekhin, A. Y., \& Pavlov, G. G. 1997, ApJ, 483, 414

Potekhin, A. Y., Chabrier, G., \& Shibanov, Yu A. 1999, Phys. Rev. E, 60, 2193

Saumon, D., Chabrier, G., \& Van Horn, H. M. 1995, ApJS, 99, 713 